\title{
Ausschreibung
}

\section{Praktiker-Preis der Deutschen Gesellschaft für Präventivzahnmedizin}

Die Deutsche Gesellschaft für Präventivzahnmedizin schreibt zum ersten Mal 2017 einen Preis für den besten Kurzvortrag eines in der Praxis tätigen Kollegen aus. Der Vortrag mit einer Länge von 15 Minuten soll bei einem wissenschaftlichen Programm der Jahrestagung vom 23. bis 25. November in Berlin gehalten werden. Ziel des mit 500 Euro dotierten Preises ist es, präventionsorientierte Handlungskonzepte in der zahnärztlichen Praxis auszuzeichnen.

Abstracts der Vorträge müssen bis zum 31. September 2017 bei carolina.ganss@dentist.med.uni-giessen.de oder Lutz@DrLaurisch.de eingereicht werden. Nähere Infos unter dgpzm.de

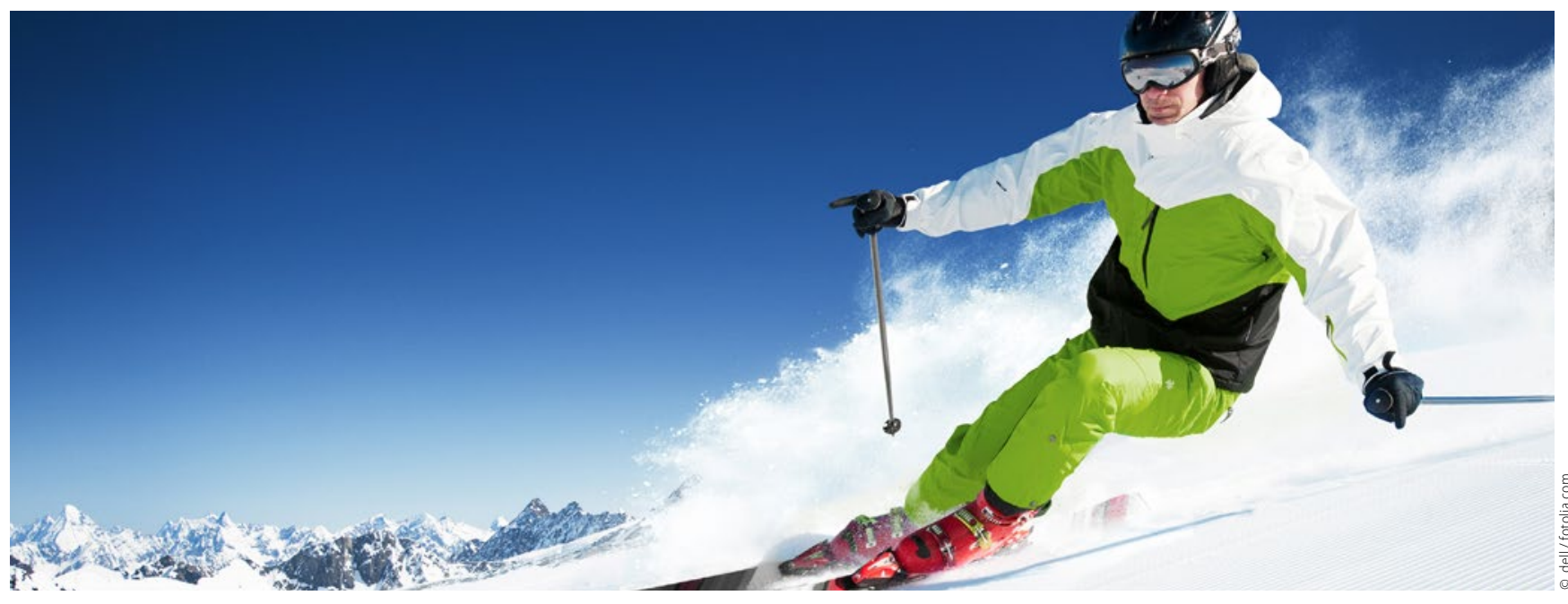

Herbert-Lewin-Preis 2017

\section{Forschungspreis zur Rolle der Ärzteschaft in der NS-Zeit}

Mit dem Herbert-Lewin-Preis werden wissenschaftliche Arbeiten zu dem Thema „Aufarbeitung der Geschichte der Ärztinnen und Ärzte in der Zeit des Nationalsozialismus“ prämiert. Die nunmehr sechste Ausschreibung wird vom Bundesministerium für Gesundheit, der Bundesärztekammer, der Kassenärztlichen Bundesvereinigung, der Bundeszahnärztekammer und der Kassenzahnärztlichen Bundesvereinigung getragen.

An der Ausschreibung teilnehmen können Zahnärzte und Ärzte sowie Psychotherapeuten als Einzelpersonen, Kooperationen oder Gemeinschaften, Studierende der Zahn- oder Humanmedizin sowie an zahn- und humanmedizinischen Fakultäten oder medizinhistorischen Instituten tätige Wissenschaftler. Jede einzelne Person und jede Arbeitsgruppe kann sich mit je einer Arbeit bewerben. Der Preis ist mit insgesamt 15.000 Euro dotiert.

Es werden nur Arbeiten berücksichtigt, die ab Januar 2012 erstellt oder veröffentlicht wurden. Die wissenschaftlichen Arbeiten müssen bis zum 15.06.2017 bei der Bundeszahnärztekammer eingegangen sein. Bewerbungsanschrift: Bundeszahnärztekammer, Hauptgeschäftsführung, Birgit Koch, Chausseestraße 13, 10115 Berlin, E-Mail: b.koch@bzaek.de

Nähere Infos unter $w w w . b z a e k . d e / F o r s c h u n g s p r e i s$.
Winterkongress 2017 in Ischgl

\section{Gemeinsam hoch hinaus in den Tiroler Alpen}

Im Kalender für das nächste Jahr sollte bei Zahnärzten und Praxismitarbeitern ein Termin nicht fehlen: Vom 19. bis 24. Februar 2017 lädt der Freie Verband Deutscher Zahnärzte (FVDZ) zum Winterkongress ins österreichische Ischgl ein. International renommierte Referenten stellen neue Behandlungskonzepte und Diagnoseverfahren vor. Themen wie Kariesprävention, Kinderzahnbehandlung und Umsetzung aktueller Hygienevorschriften in der Zahnarztpraxis stehen auf dem Programm.

Das Besondere: Auf die Teilnehmer wartet eine sechstägige Fortbildung in familiärer Atmosphäre, die Möglichkeit zum Netzwerken vor der faszinierenden Kulisse der Tiroler Bergwelt und vielfältige Freizeitmöglichkeiten. Ein weiterer Höhepunkt: Bei der Eröffnungsveranstaltung informiert der Zahnarzt und ehemalige FIFA-Schiedsrichter Dr. Markus Merk in seinem Vortrag "Sicher Entscheidungen treffen“ über ausschlaggebende Faktoren bei der Entscheidungsfindung und wie er selbst zum sicheren Entscheider wurde.

Studentischen Mitgliedern ermöglicht der Freie Verband auch in diesem Jahr wieder eine kostenfreie Teilnahme am Winterkongress sowie ein attraktives Rahmenprogramm. Insgesamt werden 20 Wochenkarten verlost. Zudem sponsert der FVDZ die Unterkunft für sechs Nächte inklusive Frühstück. Das Gewinnspiel läuft bis zum 31. Dezember. Weitere Informationen erhalten Sie unter $w w w . f v d z . d e / i s c h g l 2017$. 